\title{
Not so strictosidine
}

\author{
By Michael J. Haas, Senior Writer
}

Researchers at the Massachusetts Institute of Technology think they have overcome a significant hurdle in the biosynthesis of indole alkaloids-a family of compounds naturally produced in plants that includes marketed cancer drugs such as vinblastine and vincristine. In addition to producing intermediates for these therapeutically valuable compounds, the technique could be used to produce new alkaloids.

Alkaloid biosynthesis is typically carried out by expressing the relevant plant enzymes in microorganisms such as Escherichia coli and yeast, both of which are easier to engineer and grow than plants. But the utility of microorganisms is limited by the lack of understanding of the enzymes involved in alkaloid production in plants, as many are unidentified.

Moreover, the synthesis of pharmacologically interesting alkaloid structures in plants can involve as many as 20 enzymes-more than a single engineered microorganism can express without compromising its survival and growth. ${ }^{1}$ This limitation restricts the complexity of the alkaloid structures that can be produced.

\section{"The possibility of using the platform to produce 20 or 30 unique, patentable structures is fascinating." -Dennis Brown, ChemGenex Pharmaceuticals Ltd.}

They expressed the mutated synthase in C. roseus hairy root cell culture, a plant tissue commonly used for laboratory-scale biosynthesis of many natural products. The team found that the engineered C. roseus platform was indeed capable of converting secologanin and tryptamine into methylated, chlorinated or brominated analogs of several known indole alkaloids, such as ajmalicine, tabersonine and catharanthine.

O'Connor and Runguphan wrote that their study "sets the stage for further metabolic engineering efforts to improve the scope and practicality of unnatural product biosynthesis in plants."

\section{Growth potential}

Based on the results, the platform has the potential to explore chemical space that was not currently accessible to medicinal chemistry. The key next steps are likely to be improving the platform's yields and identifying active pharmacophores.

Joachim Stöckigt, chair of pharmaceutical biology at University Mainz - and leader of two teams that solved the strictosidine crystal structure and showed that mutant strictosidine synthases could be engineered, respectively ${ }^{3,4}$ - said the MIT team's "findings significantly expand the application of biosynthesis to the generation of novel alkaloid analogs."

He noted that the platform gave higher yields of alkaloids than the naturally occurring C. roseus plant but said the yields were still too low for commercial application.

Assuming yields could be increased and that the engineered plant system would produce stable yields, Stöckigt said it should not be difficult for companies to adopt this technology. "The hairy root culture system has been around for decades" and its production is already well engineered and well developed, he noted.

Thus, said Stöckigt, "what needs to be done is screening the alkaloid analogs for biological activity and pharmacological importance."

Dennis Brown, president and CSO of ChemGenex Pharmaceuticals Ltd., agreed that generating active alkaloid pharmacophores would be key to the platform's commercial utility.

ChemGenex's lead compound, the natural alkaloid omacetaxine, is in a Phase II/III trial to treat chronic myeloid leukemia (CML). This month, the FDA granted Orphan Drug designation to omacetaxine to treat myelodysplastic syndromes (MDS), an indication for which the compound is in Phase II testing.

"Reprogramming plants is a good platform concept" for producing alkaloids, which encompass a large number of pharmacophores that are difficult to synthesize by traditional medicinal chemistry, Brown said. "The paper provides good proof of that concept." Brown told SciBX that the platform has at least two potential applications.

First, it could provide an alternative to medicinal chemistry for the generation of alkaloid pharmacophores and their analogs. "It could be interesting if the researchers were to identify a pharmacophore of high therapeutic value but with limited analogs available - and thus access 
a wide-open chemical space with implicit value," he said.

Indeed, Brown said, the platform could lend itself to a combinatorial approach that could generate a series of compounds more easily than those structures could be synthesized one at a time via medicinal chemistry. "The possibility of using the platform to produce 20 or 30 unique, patentable structures is fascinating," he said.

Second, according to Brown, the platform could complement medicinal chemistry if it could readily make a difficult-to-synthesize intermediate structure that, once made, is amenable to modification by traditional medicinal chemistry. With such a springboard, "the chemical space they could reach is very broad," he said.

Whether as an alternative or supplement to medicinal chemistry, Brown said the commercial value of the platform will depend on whether it can produce a therapeutically useful pharmacophore or an intermediate.

"You would have to pick a pharmacophore with which people have been struggling for years, then demonstrate a breakthrough" by synthesizing it on the platform, he said.

\section{Rosy prospects}

Both Brown and Stöckigt were interested in the platform's potential to identify the other $C$. roseus enzymes that are key to the production of tabersonine, ajmalicine and catharanthine.

Knowledge of those enzymes would be useful to have, Stöckigt said, because they perform complicated rearrangements of the alkaloids' carbon structures that are not well understood by medicinal chemists. Understanding how those enzymes work might uncover completely new mechanisms and chemistry, he said.

Stöckigt added: "We just need more plant enzymes to be identified. What are their functions? What genes code for them? I don't see much development in that direction. The [Nature Chemical Biology] findings should motivate people to look more for new enzymes participating in biosynthetic pathways."

O'Connor told SciBX her group will indeed attempt to identify enzymes on the pathways to ajmalicine, tabersonine and catharanthine, using substrates that carry photoaffinity labels and tags as described in previous work by her and her colleagues. ${ }^{6,7}$
O'Connor's group has begun working with a high throughput screening lab at Vanderbilt University to evaluate the bioactivity of compounds produced by her team's platform, with the goal of identifying which structural modifications are most promising for future studies, she said.

Additionally, the group is engineering mutant strictosidine synthases that could act on and increase the yield of other tryptamine analogs. O'Connor is especially interested in improving the platform's yield of catharanthine analogs with the goal of producing vinblastine analogs.

Catharanthine reacts with vindoline to produce vinblastine in the whole C. roseus plant-but not in the hairy root culture. Thus, according to O'Connor, catharanthine analogs could be coupled to commercially available vindoline using medicinal chemistry in order to produce vinblastine analogs.

Her group is also investigating the possibility of producing vinblastine analogs by cultivating whole $C$. roseus plants from the hairy root culture that expresses mutant synthases.

O'Connor told SciBX the findings reported in Nature Chemical Biology are not patented.

Haas, M.J. SciBX 2(4); doi:10.1038/scibx.2009.126

Published online Jan. 29, 2009

\section{REFERENCES}

1. Haas, M.J. SciBX 1(30); doi:10.1038/scibx.2008.720

2. Runguphan, W. \& O'Connor, S. Nat. Chem. Biol.; published online Jan. 18, 2009; doi:10.1038/nchembio.141

Contact: Sarah O'Connor, Massachusetts Institute of Technology, Cambridge, Mass.

e-mail: soc@mit.edu

3. Ma, X. et al. Plant Cell 18, 907-920 (2006)

4. Loris, E. et al. Chem. Biol. 14, 979-985 (2007)

5. Bernhardt, P. et al. Chem. Biol. 14, 888-897 (2007)

6. Friedrich, A. et al. Tetrahedron Lett. 50, 75-76 (2009)

7. Galan, M. et al. Chem. Commun. (Camb.) 31, 3249-3251 (2007)

COMPANIES AND INSTITUTIONS MENTIONED

ChemGenex Pharmaceuticals Ltd. (ASX:CSX; NASDAQ:CSXP), Geelong, Victoria, Australia

Johannes Gutenberg University Mainz, Mainz, Germany Massachusetts Institute of Technology, Cambridge, Mass. Vanderbilt University, Nashville, Tenn. 